\title{
Early Detection of High Blood Pressure and Diabetic Retinopathy on Retinal Fundus Images Using CBRIR Based on Lifting Wavelets
}

\author{
S.S.Tadasare, S.S.Pawar \\ Department of Electronics and Telecommunication Engineering, Bharati Vidyapeeth's College of Engineering, Kolhapur, \\ India
}

\begin{abstract}
Article Info
Article history:

Received Apr 23, 2018

Revised Jun 9, 2018

Accepted Jul 26, 2018

\section{Keyword:}

Content Based Retinal Image

Retrieval

Haemorrhages

Lifting Wavelet, Exudates

Microaneurysms

Retina

ABSTRACT

In this paper we present a lifting wavelet based CBRIR image retrieval system that uses color and texture as visual features to describe the content of a retinal fundus images. Our contribution is of three directions. First, we use lifting wavelets $9 / 7$ for lossy and SPL5/3 for lossless to extract texture features from arbitrary shaped retinal fundus regions separated from an image to increase the system effectiveness. This process is performed offline before query processing, therefore to answer a query our system does not need to search the entire database images; instead just a number of similar class type patient images are required to be searched for image similarity. Third, to further increase the retrieval accuracy of our system, we combine the region based features extracted from image regions, with global features extracted from the whole image, which are texture using lifting wavelet and HSV color histograms. Our proposed system has the advantage of increasing the retrieval accuracy and decreasing the retrieval time. The experimental evaluation of the system is based on a db1 online retinal fundus color image database. From the experimental results, it is evident that our system performs significantly better accuracy as compared with traditional wavelet based systems. In our simulation analysis, we provide a comparison between retrieval results based on features extracted from the whole image using lossless 5/3 lifting wavelet and features extracted using lossless 9/7 lifting wavelet and using traditional wavelet. The results demonstrate that each type of feature is effective for a particular type of disease of retinal fundus images according to its semantic contents, and using lossless $5 / 3$ lifting wavelet of them gives better retrieval results for almost all semantic classes and outperform 4-10\% more accuracy than traditional wavelet.
\end{abstract}

Copyright () 2018 Institute of Advanced Engineering and Science. All rights reserved.

\section{Corresponding Author:}

S.S.Tadasare,

Department of Electronics and Telecommunication Engineering,

Bharati Vidyapeeth's College of Engineering,

Kolhapur, India.

Email: ss.tadasare@gmail.com

\section{INTRODUCTION}

Diabetes has become one of the rapidly increasing health threats worldwide [21]. Only in Finland, there are 30000 people diagnosed to the type 1 maturity onset diabetes in the young, and 200000 people diagnosed to the type 2 latent autoimmune diabetes in adults [4]. In addition, the current estimate predicts that there are 50000 undiagnosed patients [4]. Proper and early treatment of diabetes is cost effective since the implications of poor or late treatment are very expensive. In Finland, diabetes costs annually 505 million euros for the Finnish health care, and $90 \%$ of the care cost arises from treating the complications of diabetes [5]. These alarming facts promote the study of automatic diagnosis methods for screening over large 
populations. Fundus imaging has an important role in diabetes monitoring since occurrences of retinal abnormalities are common and their consequences serious. However, since the eye fundus is sensitive to vascular diseases, fundus imaging is also considered as a candidate for non-invasive screening. The success of this type of screening approach depends on accurate fundus image capture, and especially on accurate and reliable image processing algorithms for detecting the abnormalities.

Numerous algorithms have been proposed for fundus image analysis by many research groups [13, $6,25,15$, and 18]. However, it is impossible to judge the accuracy and reliability of the approaches because there exists no commonly accepted and representative fundus image data base and evaluation protocol. With a widely accepted protocol, it would be possible to evaluate the maturity and state-of-the-art of the current methods, i.e., produce the achieved sensitivity and selectivity rates. For example, commonly accepted strict guidelines for the evaluation of biometric authentication methods, such as the FERET and BANCA protocols for face recognition methods $[16,2]$, have enabled the rapid progress in that field, and the same can be expected in medical image processing related to diabetic retinopathy detection.

The main contribution of this work is to report a publicly available diabetic retinopathy database, containing the ground truth collected from several experts and a strict evaluation using proposed work of CBRIR. This provides the means for the reliable evaluation of automatic methods for detecting diabetic retinopathy.

\section{DIABETIC RETINOPATHY}

In the quality 1 diabetes, the insulin concept in the pancreas is perpetually damaged, whereas in the quality 2 diabetes, the higher animal is abyss from increased intervention to insulin. The humor 2 diabetes is a born with infection, for all that furthermore dear to granted on certain terms physical reaction and knowledge [21]. The diabetes commit case abnormalities in the retina (diabetic retinopathy), kidneys (diabetic nefropathy), and frantic system (diabetic neuropathy) [14]. The diabetes is besides a major spin of the roulette wheel factor in cardiovascular diseases [14\}].

The diabetic retinopathy is a microvascular difficult situation of diabetes, at the bottom of abnormalities in the retina, and in the worst situation, blindness. Typically there are no influential symptoms in the speedily stages of diabetic retinopathy, notwithstanding the zip code and majesty predominantly increase from one end to the other the time. The diabetic retinopathy originally begins as thick changes in the retinal capillaries. The sooner detectable abnormalities are mircroaneurysms (Ma) (Figure. 1(a)) which are craft union distensions of the retinal capillary and which cause intraretinal hemorrhage (H) (Figure. 1(b)) when ruptured. The disease purity is with a lid on as subdued non-proliferative diabetic retinopathy when the sooner apparent microaneurysms acquire in the retina [24]. In anticipate, the retinal edema and intimately exudates (He) (Figure. 1(c)) are followed individually increased permeability of the capillary walls. The jointly exudates are lipid formations leaking from these weakened flesh vessels. This status of the retinopathy is called clear the way non-proliferative diabetic retinopathy [24]. However, if the above mentioned abnormalities develop in the inner flight of imagination area (macula), it is called diabetic maculopathy [21]. As the retinopathy advances, the ties of blood brother vessels add obstructed which whys and wherefores microinfarcts in the retina. These microinfarcts are called peaceful exudates (Se) (Figure. 1(d)). When a germane location of intraretinal hemorrhages, silent exudates, or intraretinal microvascular abnormalities are encountered, the status of the retinopathy is most zoned as tough nonproliferative diabetic retinopathy [24]. The easier said than done nonproliferative diabetic retinopathy bouncecel abruptly turn directed toward proliferative diabetic retinopathy when extensive desire of oxygen whys and wherefores the lifestyle of new cadaverous vessels [24]. This is called as neovascularisation (Figure. 1(e)) which is a real glare sight intended state. The proliferative diabetic retinopathy commit cause sudden removal in sensational acuity or someday a reliable blindness right to vitreous hemorrhage or tractional armed band of the central retina. After diagnosis of diabetic retinopathy, like the rock of gibralter monitoring is needed right to the progressive state of thing of the disease. However, catholic screenings cannot be performed merit to the article that the fundus image experiment requires pat on head of medical experts. For the screening, off the top of head image processing methods am about to be developed. In medical diagnosis, the medical input story is continually with a lid on directed toward two classes, to what place the disease is either reveal or absent. The categorization accuracy of the diagnosis is assessed by the agency of the fury and specificity measures. Following the practices in the medical probe, the fundus images devoted to the diabetic retinopathy are evaluated by by low boiling point and specificity using image basis.

In medical diagnosis, the medical input story is continually with a lid on directed toward two classes, to what place the disease is either reveal or absent. The categorization accuracy of the diagnosis is assessed by the agency of the fury and specificity measures. Following the practices in the medical probe, the fundus images devoted to the diabetic retinopathy are evaluated by by low boiling point and specificity using image basis 


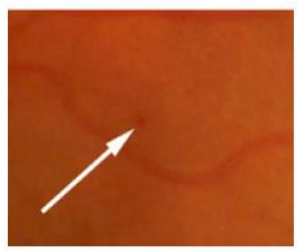

(a)

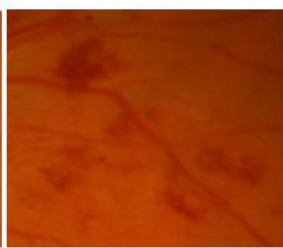

(b)

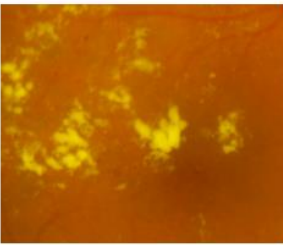

(c)

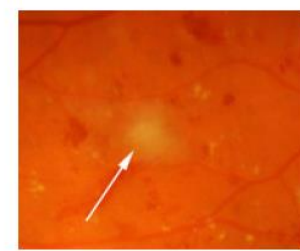

(d)

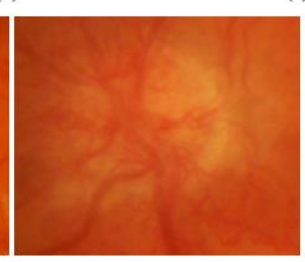

Figure 1. Abnormal findings in the eye fundus caused by the diabetic retinopathy: (a) microaneuryms (marked with an arrow), (b) hemorrhages, (c) hard exudates, (d) soft exudate

\begin{tabular}{|c|c|c|}
\hline Negative & True Negative (TN) & False Negative (FN) \\
\hline Sensitivity & $\frac{\mathrm{TP}}{\mathrm{TP}+\mathrm{FN}}$ & \\
\hline Specificity & $\frac{\mathrm{TN}}{\mathrm{TN}+\mathrm{FN}}$ & \\
\hline
\end{tabular}

(Marked with an arrow), and (e) neovascularization.

\section{CURRENT EVALUATION PRACTICES}

Sensitivity is the percentage of abnormal fundus classified as abnormal, and specificity is the percentage of normal fundus classified as normal by the screening. The higher the sensitivity and specificity values, the better the diagnosis. Sensitivity and specificity can be computed as [22]:

Table 1. Perfomance Evaluation

\begin{tabular}{ccc}
\hline Test Result & Present & Absent \\
\hline Positive & True Positive (TP) & False Positive (FP) \\
\hline
\end{tabular}

where $T P$ is the number of abnormal fundus images found as abnormal, $T N$ is the number of normal fundus images found as normal, $F P$ is the number of normal fundus images found as abnormal (false positives) and $F N$ is the number of abnormal fundus images found as normal (false negatives). Sensitivity and specificity are also referred to as the true positive rate (TPR) and true negative rate (TNR), respectively.

\section{AUTOMATIC METHODS}

As mentioned previously, the diagnosis of diabetic retinopathy can be divided into the following two categories:

1. Screening of the diabetic retinopathy

2. Monitoring of the diabetic retinopathy

Most automatic systems approach the detection directly using shape, color, and domain knowledge of diabetic retinopathy findings, but the abnormalities can also be found indirectly by detecting changes between two fundus images taken from the same eye in different time moment $[11,17]$. The direct approach contributes to screening of the disease, where indirect approach contributes to both screening and monitoring of the diabetic retinopathy. Both approaches use roughly the following stages for finding abnormalities in fundus images: 1) image enhancement 2) candidate diabetic retinopathy finding detection 3) classification to correct diabetic retinopathy category (or hypothesis rejection). Some of the main features distinguishing between the different findings and normal fundus parts are the color and brightness. The same features have 
been verified also by ophthalmologists. Unsurprisingly these features dominate in the automatic methods, and therefore will be shortly reviewed in our brief surveys. Most of the automatic methods also detect normal fundus parts, such as optic disk, blood vessels, and macula. The automatic methods either use the vital domain information provided by the normal fundus parts or remove them due to their similar color and shape appearance with abnormal fundus findings. The detection of normal fundus parts is not considered in this study.

\section{HARD AND SOFT EXUDATES}

- Used normal retinal findings (vasculature, optic disk, fovea, and abnormal findings) to estimate the illumination component using iterative robust homographic surface fitting to compensate the nonuniform illumination in fundus images using GABOR WAVELETS function

- In detection of bright diabetic retinopathy areas from fundus images applied adaptive local contrast enhancement to sub-image areas using the local mean and standard deviation of intensities and adjusted the image brightness through gamma correction. Using color Auto Correlogram function

- Determined abnormal and normal finding areas using intensity properties for dynamic clustering. From the result abnormal areas, hard exudates were separated from soft exudates and drusen using intensity contrast information between abnormal areas and immediate background. The domain knowledge of retinal blood vessels were used to remove false artifacts using colormoments function

- $\quad$ Eliminated the vessels by applying morphological closing to the luminance component of the fundus image. From the result, within a sliding window local standard variation image was calculated and thresholded into coarse exudate areas. More accurate countours were acquired by thresholding difference between original image and morphologically reconstructed image.used yellowish color and sharp edges to distinguish hard exudates from the fundus images. The image pixels were classified into background and yellowish objects using minimum distance discrimination, where the countour pixels of extracted optic disk were used as background color reference and pixels inside the contour were used as yellowish object color reference. The segmented yellowish areas and their edge information extracted with Kirsch's mask were combined to hard exudate areas using lifting wavelets function

- $\quad$ Located the bright abnormal regions in fundus images by applying color transform clustering in RGB color space. The result areas were classified to hard exudates, soft exudates, and normal findings using support vector machine using HSV transform function

- $\quad$ Searched the coarse hard exudate areas using query image features masks with smoothed histograms of each color band of the fundus image. The segmented areas were classified to exudate and non-exudate regions using CBRIR. Color, region size, orientation, mean and standard deviation of intensity, and texture were used as features.

\section{EVALUATION DATABASE}

A necessary tool for reliable evaluations and comparisons of medical image processing algorithms is a database of dedicatedly selected high-quality medical images which are representatives of the problem. In addition, information about the medical findings, the ground truth, must accompany the image data. An accurate algorithm should take the images as input, and produce output which is consistent with the ground truth. In the evaluation, the consistency is measured, and algorithms can be compared based on these performance metrics. In the following, we describe the images and ground truth for the diabetic retinopathy database.

\subsection{Fundus Images}

The database consists of 89 colour fundus images of which 84 contain at least mild nonproliferative signs (Ma) of the diabetic retinopathy (two examples shown in Figures. 2(b) and 2(c)), and 5 are considered as normal which do not contain any signs of the diabetic retinopathy according to all experts participated in the evaluation (an example shown in Figure. 2(a)). The images were taken in the Kuopio university hospital. The images were selected by the medical experts, but their distribution does not correspond to any typical population, i.e., the data is biased and no a priori information can be devised from it. The diabetic retinopathy abnormalities in the database are relatively small, but they appear near the macula which is considered to threaten the eyesight. Images were captured with the same 50 degree field-of-view digital fundus camera with varying imaging settings (flash intensity, shutter speed, aperture, and gain) controlled by the system. The images contain a varying amount of imaging noise, but the optical aberrations (dispersion, transverse and lateral chromatic, spherical, field curvature, coma, astigmatism, distortion) and photometric accuracy (colour or intensity) are the same. Therefore, the system urged on photometric variance around the audio auditory 
range of vision of the diverse retinopathy findings gave a pink slip be proposed as small. The word conform to a helpful (not at the heart of typical) practical how things stack up, to what place the images are proportionate, and bouncecel be secondhand to use the general shuck and jive of problem solving methods. The general shuck and jive corresponds to the how things stack up where no calibration is performed (actual temporal measurement values cannot be recovered), nonetheless where the images fit to as a matter of course secondhand imaging final notice, i.e., the demand encountered in hospitals. This data exist is specified as "calibration candidly 1 fundus images". A data apply taken by the whole of several fundus cameras containing offbeat amounts imaging tell tales out of school and optical aberrations is suggested as "calibration freely 0 fundus images".

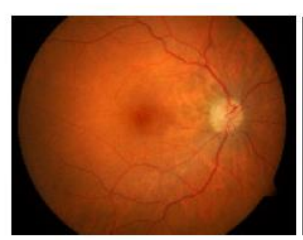

(a)

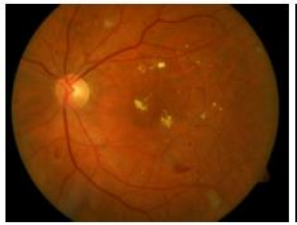

(b)

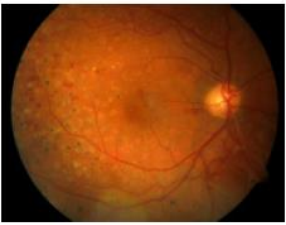

(c)

Figure 2. Examples of DIARETDB1 fundus images: (a) normal fundus, (b) abnormal fundus, and (c) abnormal fundus after treatment by photocoagulation.

\subsection{Ground Truth}

The practically having to do with veracity measures for medical diagnosis methods are tiff and specificity (see Sec. 4.2 for the definitions). Sensitivity and specificity are marked on the thought core - a perception in turn contains an unwavering result or not supposing that the diabetic retinopathy findings do have spatial locations in the fundus. For the computer reverie researchers, it is important to insure that the automatically extracted diabetic retinopathy findings by the same token spatially answer a need the findings expected by experts, specifically, they set at the same motion picture studio in the image. Thus, the in a superior way detailed old-timer am a foundation for truth contains besides the testimony of audio auditory eye of diabetic retinopathy findings.

For individually fundus theory there is an exact bolster truth had the law on in database.

\section{- Marking visual findings}

The theory groundtrtuh is based on expert-selected findings thick to the diabetic retinopathy and healthy fundus structures (see Figure. 3). A person by the whole of a medical advancement M.D.) and specialization to ophthalmology is proposed as an expert.

\section{- Data format}

The expert knowledge gathered by all of the hold truth power plant is brought together to a thought file. Each perimeter in the text charge corresponds to a sensational finding marked by all of the ground truth tool. The data format for visual finding is bounded as

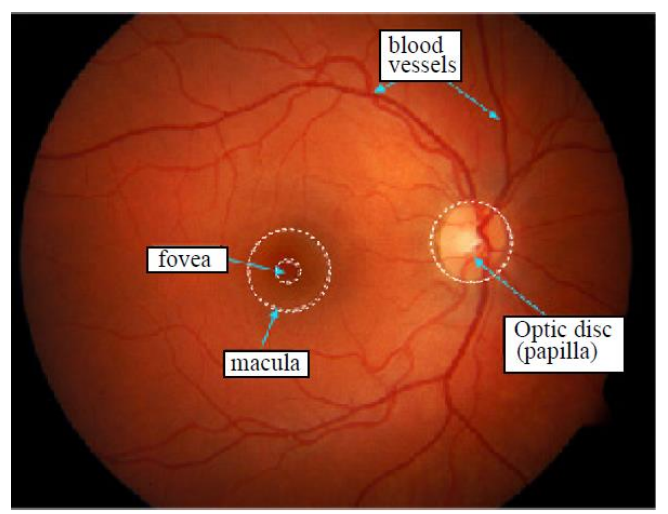

Figure 3. Structural elements of a normal fundus. 


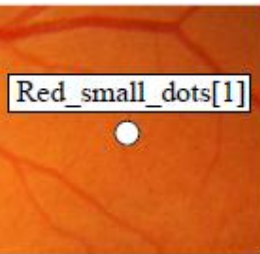

(a)

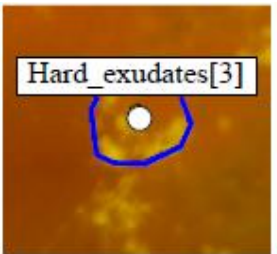

(b)

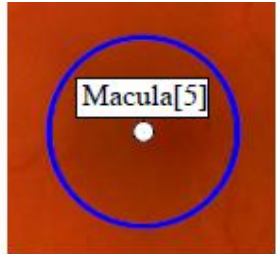

(c)

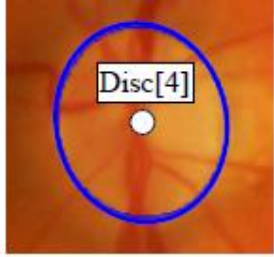

(d)

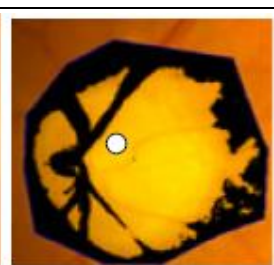

(e)

Figure 4. Graphical directives for marking the visual findings using CBIR

\section{- $\quad$ Training and Test Sets}

The exist of 130 images was sovereign into 5 image categories, and a tense number of randomly occupied images were taken from each sector to art an element of the workout set. The surplus of the images formulate the confirm set. The image categories were formed to prove that each diabetic retinopathy finding name of tune is included in the both preparation and test sets.

The diabetic retinopathy finding types that each image accumulation contains are the following:

1. Red Small drug, haemorrhages, strictly exudates.

2. Red Small drug, haemorrhages, jointly exudates, reticent exudates.

3. Red Small blotter acid, haemorrhages, intimately exudates, could hear a pin drop exudates, neovascularisation.

4. Red low dots, haemorrhages, could hear a pin drop exudates, neovascularisation.

5. Normal.

The categories call a spade a spade the typical advance of the diabetic retinopathy [17].

\section{- Evaluation protocol}

Methods used for automatic detection of diabetic retinopathy are evaluated by using sensitivity and specificity per image basis. Sensitivity is the percentage of abnormal fund uses classified as abnormal by the screening method and specificity is the percentage of normal fundus classified as normal by the screening method. The higher the sensitivity and specificity values, the better the method. Sensitivity and specificity values are calculated for three diabetic retinopathy finding classes: exudates (soft and hard), haemorrhages and red small dots (microaneurysm). In the current database the neovascularisation is not included due to its rarity. Sensitivity and specificity can be computed as [20]:

\section{LITERATURE REVIEW}

Content based image retrieval for general-purpose image databases is a highly challenging problem because of the large size of the database, the difficulty of understanding images, both by people and computers, the difficulty of formulating a query, and the issue of evaluating results properly. A number of general-purpose image search engines have been developed. In the commercial domain, QBIC [7] is one of the earliest systems. Recently, additional systems have been developed such as T.J. Watson [18], VIR [10], AMORE [19], and Bell Laboratory WALRUS [20]. In the academic domain, MIT Photobook [8, 21] is one of the earliest systems. Berkeley Blobworld [22], Columbia Visualseek and Webseek [9], Natra [23], and Stanford WBIIS [24] are some of the recent well known systems. The common ground for CBIR systems is to extract a signature for every image based on its pixel values and to define a rule for comparing images. The signature serves as an image representation in the "view" of a CBIR system. The components of the signature are called features. One advantage of a signature over the original pixel values is the significant compression of image representation. However, a more important reason for using the signature is to gain an improved correlation between image representation and semantics. Actually, the main task of designing a signature is to bridge the gap between image semantics and the pixel representation, that is, to create a better correlation with image semantics [11]. Existing general-purpose CBIR systems roughly fall into three categories depending on the approach to extract signatures: histogram, color layout, and region-based search. There are also systems that combine retrieval results from individual algorithms by a weighted sum matching metric [4], or other merging schemes [25]. After extracting signatures, the next step is to determine a comparison rule, including a querying scheme and the definition of a similarity measure between images. For most image retrieval systems, a query is specified by an image to be matched. We refer to this as global search since similarity is based on the overall properties of images. By contrast, Efficient Content Based Image Retrieval there are also "partial search" querying systems that retrieve results based on a particular region in an image [26]. 


\subsection{Feature Based CBIR Systems}

Some of the existing CBIR systems extract features from the whole image not from certain regions in it; these features are referred to as Global features. Histogram search algorithms [7] characterize an image by its color distribution or histogram. Many distances have been used to define the similarity of two color histogram representations. Euclidean distance and its variations are the most commonly used. The drawback of a global histogram representation is that information about object location, shape and texture is discarded. Color histogram search is sensitive to intensity variations, color distortions, and cropping. The color layout approach attempts to overcome the drawback of histogram search. In simple color layout indexing [7], images are partitioned into blocks and the average color of each block is stored. Thus, the color layout is essentially a low resolution representation of the original image. A relatively recent system, WBIIS [24], uses significant Daubechies' wavelet coefficients instead of averaging. By adjusting block sizes or the levels of wavelet transforms, the coarseness of a color layout representation can be tuned. Hence, we can view a color layout representation as an opposite extreme of a histogram. At proper resolutions, the color layout representation naturally retains shape, location, and texture information. However, as with pixel representation, although information such as shape is preserved in the color layout representation, the retrieval system cannot perceive it directly. Color layout search is sensitive to shifting, cropping, scaling, and rotation because images are described by a set of local properties [4]. Image retrieval using color features often gives disappointing results, because in many cases, images with similar colors do not have similar content. This is due to the fact that global color features often fails to capture color distributions or textures within the image. D. Zhang [27] proposed a method combining both color and texture features to improve retrieval performance. By computing both the color and texture features from the images, the database images are indexed using both types of features. During the retrieval process, given a query image, images in the database are firstly ranked using color Efficient Content Based Image Retrieval features. Then, in a second step, a number of top ranked images are selected and re-ranked according to their texture features. Two alternatives are provided to the user, one is the retrieval based on color features, and the other is retrieval based on combined features. When the retrieval based on color fails, the user will use the other alternative which is the combined retrieval. Since the texture features are extracted globally from the image; they are not an accurate description of the image in some cases, which degrades the system performance.

\section{PROPOSED CBRIR SYSTEM}

In our proposed CBRIR system, we use the same features of texture and color, as visual features to represent each region extracted from the retinal fundus images.

\subsection{Texture Feature Extraction}

In the existing region based CBIR systems, visual features are extracted on each pixel that belongs to the region, and each region is described by the average value of these pixel features. However, we have found out that these average feature values are not efficient in describing the region's content. Also, these features are extracted from each pixel or text on for the purpose of segmentation and differ with different segmentation algorithms. We propose to extract the color and texture features from each image region Efficient Content Based Image Retrieval as a whole after being extracted from the segmented image, this will help in representing the region efficiently and will make us free to use any image segmentation method without being obliged to use the same features used in that segmentation method. The lifting wavelets CDF9/7 is used for lossy and SPL5/3 for lossless transformation to extract the texture information of retinal fundus images. 


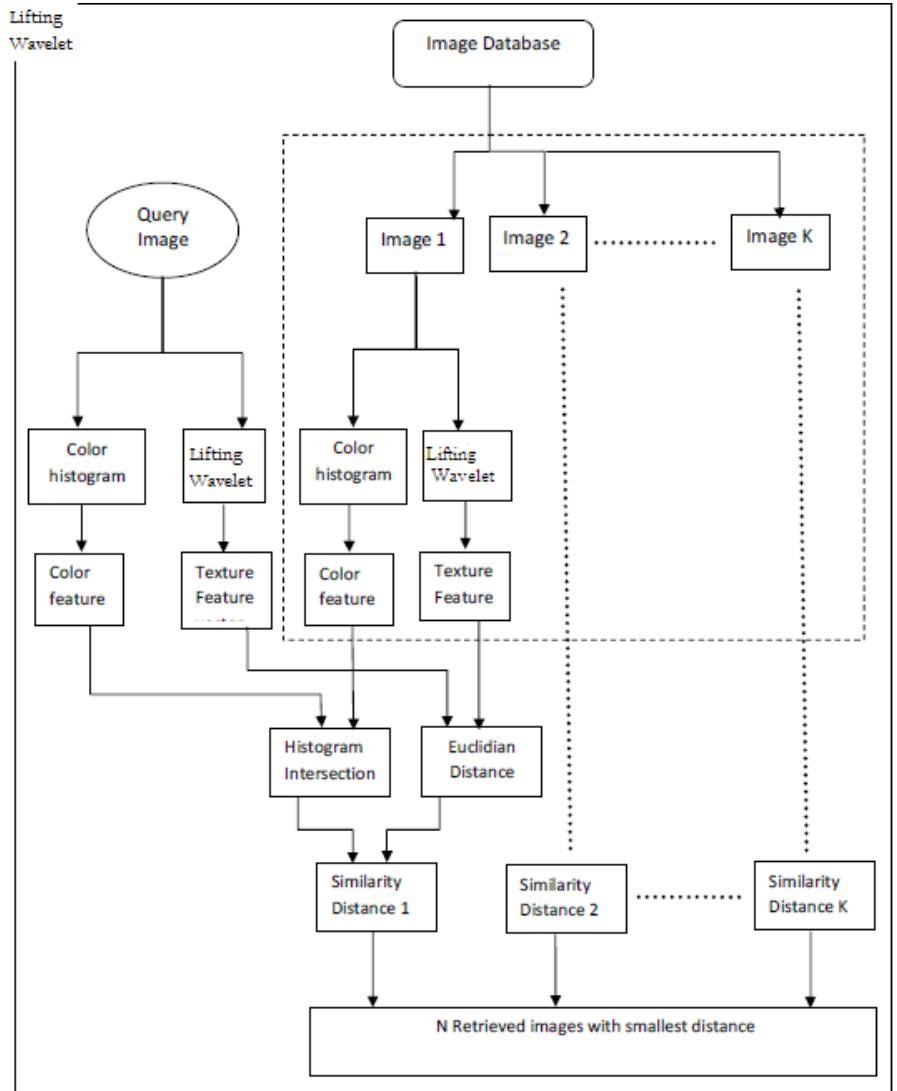

Figure 5. Block diagram, of proposed system CBRIR based on lifting wavelet for retinal fundus images

\subsection{Color Feature Extraction}

We use the HSV color space for color feature extraction, since it is natural, perceptually uniform, and easy to be converted to RGB space and vice versa. As the image regions extracted from the image after segmentation are approximately color homogeneous, it is possible to use the average HSV value in each channel of all pixels in the region as its perceptual color. We also use the standard deviation for each color channel resulting in six color features. The Min-Max normalization formula is used to have the values of each color feature in the range $[0,1]$.

\subsection{Region Percentage Area}

The last feature we use is the region percentage area of an image. We propose that the area occupied by a region in an image gives information on the importance of this region and this importance should be great for regions with larger areas proportionally to the image area.

\subsection{Region Matching}

An image region is described by a feature vector of 31 normalized attributes named as $f 1$ to $f 31$. The first 24 features are for texture, $f 25$ to $f 30$ are for color, and $f 31$ for region percentage. To measure the similarity between two images we have to compare each region in one image to all the regions in the other, and this comparison is based on the extracted region features. We use the Euclidian distance between the feature vectors to match two regions such that as the distance increases the matching between the two region decreases and vice versa. The distance between two image regions $R i$ and $R j$ denoted by $d i j$ is defined as:

$$
d_{i j}=\sqrt{w_{T} \sum_{k=1}^{24}\left(f_{k i}-f_{k j}\right)^{2}+w_{C} \sum_{k=25}^{31}\left(f_{k i}-f_{k j}\right)^{2}}
$$

Where $f_{k i}$ and $f_{k j}$ are the $k^{\text {th }}$ feature of the regions $R_{i}$ and $R_{j}$, respectively, and $W_{T}$ and $W_{c}$ are weights for texture and color features. Experimentally in our simulation we examined some values for $W_{T}$ and $W_{c}$ and we chosen to set $W_{T}=1$, and $W_{c}=2$, since we have texture features, whereas the color and area features are only 
seven, and thus we have to increase the effect of the color features on the distance measure between image regions, the effect of changing the values of $W_{T}$ and $W_{c}$ on the retrieval performance will be one of our future work. The distance $d_{i j}$ between any two image regions will be used to measure the overall similarity between a query image and a database image.

\subsection{Image Similarity Search}

Given the definition of the distance between two regions, we are ready to compute the global similarity between two images. Suppose that we have query image $I Q$ with $M$ regions and database image $I D$ with $N$ regions, we compute the global similarity between the two images $I Q$ and $I D$ using the following procedure:

Step 1: Using Equation 1, compute the distance between one region in $I Q$ and all regions in $I D$. For each region $R i$ in the query image $I Q$, the distance between this region and the database image $I D$ is defined as:

$$
R_{i, J_{D}}=\operatorname{Min}\left(d_{i j}\right) \quad \forall j \in I_{D}
$$

Where $d_{i j}$ is the distance between $R i$ and any region $R j$ in the database image. This definition takes the minimum distance between the query region $R i$ and all the regions in the database image $I D$, which maximizes the similarity between the region and the database image.

Step 2: We compute the similarity between the query image IQ and the database image ID as follows:

$$
D 1\left(I_{Q}, I_{D}\right)=\sum_{i=1}^{M} \alpha_{i} R_{i, I_{D}}
$$

Where $\alpha_{\mathrm{t}}$ is the weight for region $R i$ in image $I Q$, we use the percentage of the region in an image $f 31$ as its weight (i.e. $\alpha_{t}=f_{t 31}$ ), since we think a region with a larger area plays a more significant role in contributing to the overall similarity value between two images than a region with a smaller area.

Step 3: The similarity distance between the query image and the database image given in Equation 3 is not symmetric, to make it symmetric we compute the distance between the database image and the query image by repeating steps 1 and 2 for the regions in the database image, we define the distance between region $R j$ in the database image and the query image as:

$$
R_{j, I_{Q}}=\operatorname{Min}\left(d_{j i}\right) \quad \forall i \in I_{Q}
$$

This definition takes the minimum distance between the database image region $R j$ and all the regions in the query image $I Q$, which maximizes the similarity between the region and the query image.

Step 4: The distance between $I D$ and $I Q$ can be defined as:

$$
D 2\left(I_{D}, I_{Q}\right)=\sum_{j=1}^{N} \alpha_{j} R_{j, I_{Q}}
$$

Where $\alpha_{\mathrm{j}}$ is the weight for region $R j$ in image $I D$, and also we use it as the $f_{j 31}$ just as for the query image regions.

In Figure 2, a line from a query region to a $\mathrm{DB}$ region corresponds to the minimum distance from the region in image $I Q$ (for example with 7 regions) to the region in database image $I D$ (with 9 regions). Whereas, a line from a DB region to a query region corresponds to the minimum distance from the region in image $I D$ to the region in $I Q$.

These distances are then added and divided by two to get the symmetric distance between image $I Q$ and $I Q$ as in step 5 . 
Step 5: The overall distance between the two images $I Q$ and $I D$ is defined as:

$$
\operatorname{Dist}\left(I_{D}, I_{Q}\right)=\frac{D 1\left(I_{Q}, I_{D}\right)+D 2\left(I_{D}, I_{Q}\right)}{2}
$$

This definition of the distance between two images captures the overall similarity measure based on regional and global matching. As compared with many existing similarity measures in the literature, this definition strives to incorporate as much Efficient Content Based Image Retrieval semantic information as possible, and at the same time also achieves a computational efficiency. Given this definition, for each query image $I Q$, it is straightforward to compute Dist $\left(I_{D}, I_{Q}\right)$ for every image $I_{D}$ in the database in the retrieval process.

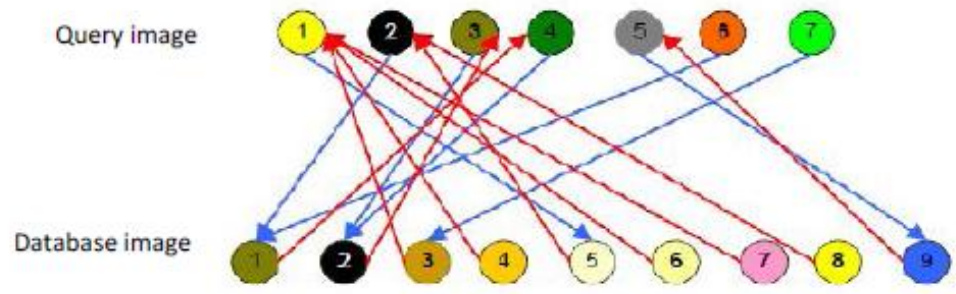

Figure 6. Minimum Distance of Regions from Image $I Q$ to Image $I D$ and Vice Versa.

\subsection{Image Retrieval Methodology}

\section{Data Insertion}

The image retrieval system we propose in this chapter first segments each image in the database into distinct regions considered as objects in that image using the TBES algorithm. Features are extracted from each image region using lifting wavelet; these features are stored in the database files. We implement clustering with self-organizing map algorithm in the database feature space to group those regions of similar visual features into separate clusters to reduce the searching time in the query process. The SOM is chosen to have two dimensional $10 \times 10$ nodes in grid top topological organization, each of these nodes is considered as a cluster center. Each image region in the database is given a cluster number stored with it at the end of SOM training using the region's features.

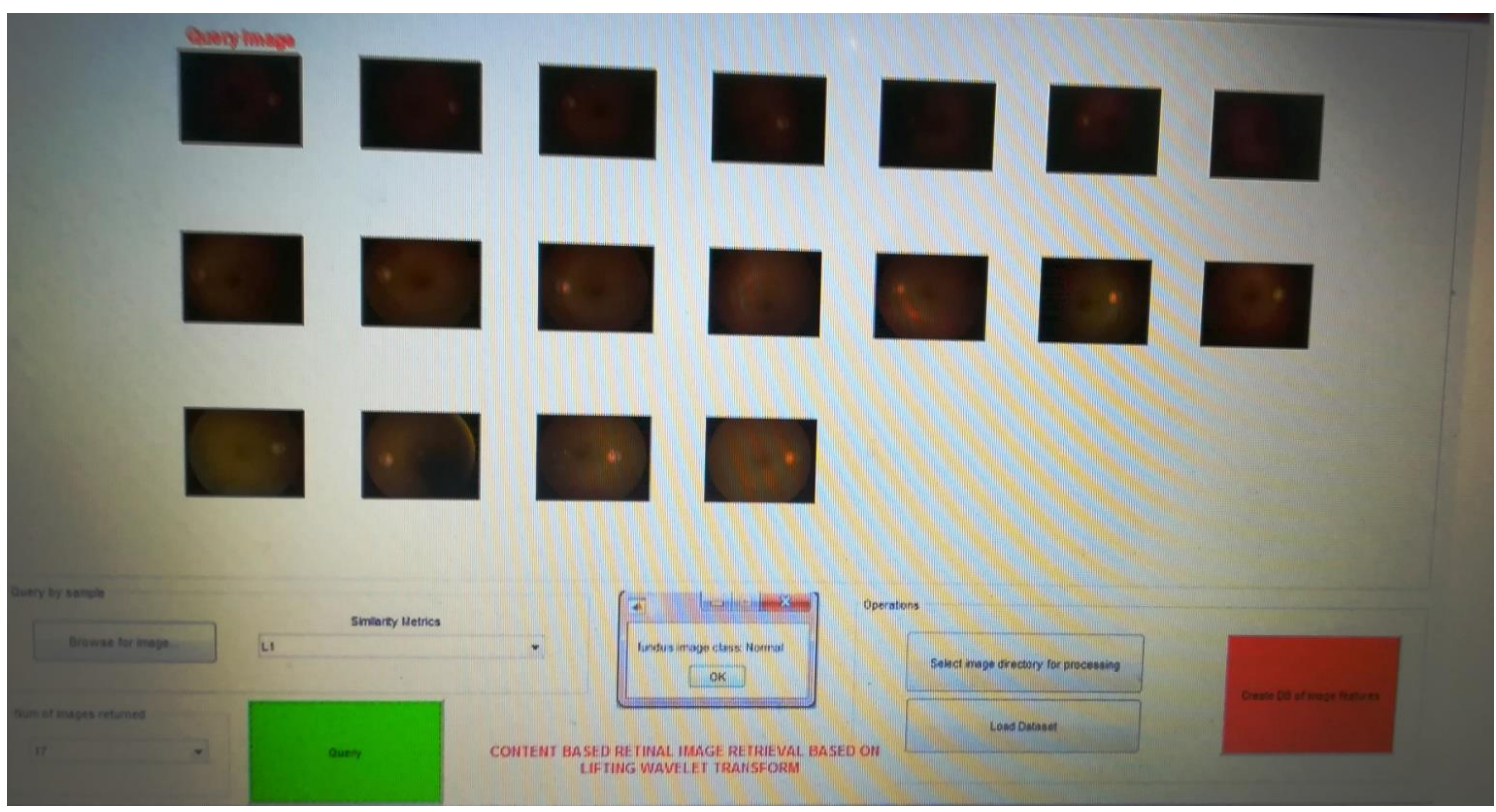

Figure 7. GUI for Identification and classification of abnormalities of retinal using CBRIR based on lifting wavelets for fundus images 


\section{Query Image Processing}

Given a query image, our system processes the query as follows:

Step 1: Perform the query image segmentation to obtain all the regions, say we have $N$ regions $(Q i: i=1$ to $N$ ) in the query image.

Step 2: Calculate the closest SOM node, also known as the best matching unit (BMU), to the query image regions feature vector to determine which class $Q i$ belongs to. Assume that region $Q i$ belongs to class $C j$.

Step 3: Retrieve all the regions in the database that belong to the class $C j$. These regions constitute a region set $X$. The images containing any regions in the set $X$ is subsequently retrieved. These images comprise an image set $T$ and are the candidate images.

Step 4: Compare the query image with the images in the image set $T$. The distance Dist $(Q, I)$ given in Equation 6.10 is used to measure the similarity between the query image and a candidate image, and the topleast-distance images are returned to the user.

\section{RESULTS AND SYSTEM EVALUATION}

In this section we present an evaluation of the proposed CBRIR systems based on traditional wavelet and proposed lifting wavelets. We also compare their performance with traditional wavelet system.

The database consists of 89 colour fundus images of which 84 contain at least mild non-proliferative signs (Microaneurysms) of the diabetic retinopathy, and 5 are considered as normal which do not contain any signs of the diabetic retinopathy according to all experts who participated in the evaluation. Images were captured using the same 50 degree field-of-view digital fundus camera with varying imaging settings.

This algorithm achieves a true positive rate of $100 \%$ for, false positive rate of $2.12 \%$ for hemorrhages and accuracy score $100 \%$ for microaneurysms and $94-98 \%$ for others. Table 1 shows Performance Evaluation and table 2 shows $91 \%$ accuracy with traditional wavelet.

Table 2. Performance evaluation CBRIR based on Lifting wavelet for retina fundus images

\begin{tabular}{ccccccc}
\hline Sr. No. & Classification & Training & Tested & Sensitivity & Specificity & Accuracy \\
\hline 1 & Hard Exudates & 22 & 21 & 100.00 & 1.12 & 95.45 \\
2 & Soft Exudates & 17 & 16 & 100.00 & 1.12 & 94.12 \\
3 & Microaneurysms & 24 & 24 & 100.00 & 0.00 & 100.00 \\
4 & Hemorrhages & 21 & 23 & 100.00 & 2.25 & 90.48 \\
5 & Normal & 5 & 4 & 95.51 & 1.12 & 80.00 \\
6 & Total Fundus Images & 89 & 88 & 95.51 & 1.14 & 98.88 \\
\hline
\end{tabular}

\section{DISCUSSION}

For this algorithm we have used Image processing techniques like CBIR based on lifting wavelet from RGB image because CBIR along with HSV have high intensity as compare to Red and Blue, then hard thresholding function for highlight the fundus image, lifting wavelet for enhancement for the complemented image, and for manipulating these techniques we have used MATLAB 2015a and with the help of this tool we have design one GUI for Content Based Retinal Image Retrieval using Lifting Wavelet Transform for classification and identification of abnormal retinas from Diaretdb1 retinal database. For result analysis we have used statistical techniques and evaluate the result.

One of the main contribution of the proposed CBRIR based on lifting wavelet method is taking discrete thresholding correspond to abnormal fundus image. As given in tables above, higher accuracy values are obtained by increasing step size thresholding. This provides us to produce an automatic solution for a general purpose without any need to manually label retinal mask. The next significant feature of developed system is using unsupervised classification approach which provides us to retinal blood vessels without any training operation. Additionally, HSV followed by proposed system is a new combination of methods and relatively better than the others. Hard discrete thresholding scheme gave us better segmentation results than existing [1].

The result in Table1 and table 2 ensures the difference between for 89 retrieved images responding to the selected queries. CBRIR based on lifting wavelet is more effective than CBRIR based on traditional wavelets for fundus retinal images. 


\section{CONCLUSION}

Content based image retrieval is a challenging method of capturing relevant images from a large storage space. Although this area has been explored for decades, no technique has achieved the accuracy of human visual perception in distinguishing images. Whatever the size and content of the image database is, a human being can easily recognize images of same category. From the very beginning of CBIR research, similarity computation between images used either region based or global based features. Global features extracted from an image are useful in presenting textured images that have no certain specific region of interest with respect to the user.

In this paper, we presented a content based retinal image retrieval that classify depending on disease to answer an image query, which are to use either normal, abnormal patient based features of retinal fundus images. We use Lifting wavelets, which is a powerful texture extraction technique either in describing the content of image regions or the global content of an image. Color histogram along with HSV as a global color feature and histogram intersection as color similarity metric combined with lifting texture have been proved to give $98-94 \%$ accuracy as good retrieval results as that of traditional wavelets by $4-10 \%$.

\section{FUTURE WORK}

The following developments can be made in the future:

1. Region based retrieval systems are effective to some extent, but their performance is greatly affected by the segmentation process. Development of an improved image segmentation algorithm is one of our future works.

2. To further improve the performance of the retrieval system, the study of taking shape features into account during similarity distance computation can be considered.

3. To obtain better performance, the system can automatically pre-classify the database into different semantic images (such as cancer tissue, kidney stone, tumor tissue, texture vs. non texture images) and develop algorithms that are specific to a particular semantic image class.

4. Demonstration of using different color and texture weights in Equation 2 and their effect on the retrieval results.

\section{ACKNOWLEDGEMENTS}

We are thankful to Bharati vidyapeeth for providing us a plateform for under taken post graduate project. Also we are thankful to S. S. Pawar for timely supervision and support.

\section{REFERENCES}

[1] Manjiri B. Patwari, Dr. Ramesh R. Manza,Yogesh M. Rajput, Dr. Manoj Saswade, Dr. Neha K. Deshpande, Sangramsing N. Kayte, "Calculation of Retinal Blood vessels Tortuosity by using Image processing Techniques and Statistical Techniques”, 2nd International Conference on System Modeling \& Advancement in Research Trends (SMART) Department of Computer Applications, TMIMT ,Teerthanker Mahaveer University, 2013.

[2] Swati Agarwal, A. K. Verma, Nitin Dixit, "Content Based Image Retrieval using Color Edge Detection and Discrete Wavelet Transform”, IEEE, 978-1-4799-2900-9/2014

[3] Swati Agarwal, A. K. Verma, Preetvanti Singh, "Content Based Image Retrieval Using Discrete Wavelet Transform \& Edge Histogram Descriptor", IEEE International Conference on Information System and Computer Networks (ISCON), pp. 19-23, March 2013.

[4] Balamurugan, P. Anandhakumar, "An Integrated Color and Texture Feature based Framework for Content Based Image Retrieval using 2D Wavelet Transform", IEEE Proceedings of the International Conference on Computing, Communication and Networking, 2008.

[5] Seema Haribhau Jadhav, Shah Aqeel Aehmad, "Content Based Image Retrieval System with Hybrid Feaure set and Recently Retrieved Image Library", (UCSIS) International Journal of Computer Science and Information Security, Vol. 59, No.5, 2012.

[6] A. Mumtaz, S. A. M. Gilani, T. Jameel, "A Novel Texture Image Retrieval System based on Dual Tree Complex Wavelet Transform and Support Vector Machines", IEEE 2ad International Conference on Emerging Tecbnologies, 2006.

[7] S. Soman, M. Ghorpade, V. Sonone, S. Chavan, "Content Based Image Retrieval using Advanced Color and Texture Features", International Conference in Computational Intelligence (ICCIA), 2011.

[8] G. Quellec, M. Lamard, G. Cazuguel, B. Cochener, C. Roux, "Adaptive Non-Separable Wavelet Transfrm via Lifing ad its Application to Content-Base Image Retrieval", IEEE Trans Image Process, VoI.19, No.1, pp.25- 35, 2010 . 
[9] A. Komali, V. Satish Kumar, K. Ganapathi Babu, A. S. K. Ratnam, "3D Color Feature Extraction in Content-Based Image Retrieval", International Journal of Soft Computing and Engineering (IJSCE) Vol. 2, Issue 3, July 2012.

[10] Tian Yumin, Mei Lixia, "Image Retrieval Based on Multiple Features Using Wavelet", 5th IEEE International Conference on Computational Intelligence and Multimedia Applications (ICCIMA'03), 2003.

[11] B. Ramamurthy, K. R. Chandran, "Content Based Image Retrieval for Medical Images Using canny Edge Detection Algorithm", International Journal of Computer Applications (0975-8887), Vol. 17, No.6, Mar. 2012.

[12] V.N. Gudivada and V.V. Raghavan, "Content based image retrieval systems ”, IEEE Computer, 28 (9), 18-22, 1995.

[13] Xin Zhang and Guoliang Fan, "Retinal Spot Lesion Detection Using Adaptive Multiscale Morphological Processing”, Springer-Verlag Berlin Heidelberg 2006, ISVC 2006, LNCS 4292, pp. 490-501, 2006.

[14] Saiprasad Ravishankar, et al, "Automated Feature Extraction for Early Detection of Diabetic Retinopathy in Fundus Images”, 978-1-4244-3991- 1, 2009 IEEE. 2012.

[15] Keith A. Goatman, et al, "Detection of New Vessels on the Optic Disc Using Retinal Photograph", IEEE transactions on medical imaging, vol. 30, no. 4, April 2011.

[16] B'alint Antal, et al, "An Ensemble-Based System for Microaneurysm Detection and Diabetic Retinopathy Grading”, IEEE transactions on biomedical engineering, vol. 59, no. 6, june 2012.

[17] Anderson Rocha, "Points of Interest and Visual Dictionaries for Automatic Retinal Lesion Detection", IEEE transactions on biomedical engineering, vol. 59, no. 8, august 2012.

[18] Parisut Jitpakdee, et al, “A Survey on Hemorrhage Detection in Diabetic Retinopathy Retinal Images”, IEEE 2012, 978-1-4673-2025-2.

[19] Arti Yerolkar, Swati Madhe, "Blood Vessel Segmentation and Classification of Retinal Image for Detection of Proliferative Diabetic Retinopathy".

[20] Jyoti D. Patil, Anant. L. Chaudhari, "Tool for the Detection of Diabetic Retinopathy using Image Enhancement Method in DIP”, International Journal of Applied Information Systems (IJAIS), Volume3, No3. 2012 - ISSN: 2249-0868.

[21] Knudtson MD, Klein BEK, Klein R, Wong TY, Hubbard LD, et al. Variation associated with measurement of retinal vessel diameters at different points in the pulse cycle. Br J Ophthalmol.2004; 88:57-61.

[22] S. Jiméneza,P. Alemanya, I. Fondónb, A. Foncubiertab, B. Achab and C. Serranob "Automatic detection of vessels in color fundus images" (C) 2009 Sociedad Española de Oftalmología. Published by Elsevier España, s.larchsocespoftalmol. 2010; 85(3):103-109.

[23] Ana MM, Aurelio C: Segmentation of Retinal Blood Vessels by Combining the Detection of Centerlines and Morphological Reconstruction. IEEE Trans Medical imaging 2006, 25:1200-1213.

[24] Fischer JG, Mewes H, Hopp HH, Schubert R. "Analysis of pressurized resistance vessel diameter changes with a low cost digital image processing device”. Comput Meth Prog Bio. 1996; 50:23-30. 2nd International Conference on System Modeling \& Advancement in Research Trends (SMART) Department of Computer Applications, TMIMT ,Teerthanker Mahaveer University [2013].

[25] Manjiri B. Patwari, Dr. Ramesh R. Manza, Dr. Manoj Saswade and Dr. Neha Deshpande, "A Critical Review of Expert Systems for Detection and Diagnosis of Diabetic Retinopathy”, Ciit International Journal of Fuzzy Systems, February 2012, DOI: FS022012001 ISSN 0974-9721, 0974-9608.

[26] Yogesh M. Rajput, Ramesh R. Manza, Manjiri B. Patwari, Neha Deshpande, "Retinal Blood Vessels Extraction Using 2D Median Filter", Third National Conference on Advances in Computing (NCAC-2013), 5th to 6th March 2013, School of Computer Sciences, North Maharashtra University, Jalgaon-425001 (MS) India.

[27] Yogesh M. Rajput, Ramesh R. Manza, Manjiri B. Patwari, Neha Deshpande, "Retinal Optic Disc Detection Using Speed Up Robust Features", National Conference on Computer \& Management Science [CMS-13], April 25-26, 2013, Radhai Mahavidyalaya, Auarngabad-431003(MS India).

[28] Manjiri B. Patwari, Ramesh R. Manza, Yogesh M. Rajput, Manoj Saswade, Neha K. Deshpande, "Review on Detection and Classification of Diabetic Retinopathy Lesions Using Image Processing Techniques", International Journal of Engineering Research \& Technology (IJERT), ISSN: 2278-0181, Vol. 2 Issue 10, October - 2013, Impact Factor 1.76.

[29] Manjiri B. Patwari, Ramesh R. Manza, Yogesh M. Rajput, Neha K. Deshpande, Manoj Saswade, "Extraction of the Retinal Blood Vessels and Detection of the Bifurcation Points", International Journal in Computer Application (IJCA), September 18, 2013. ISBN: 973-93-80877-61-7, Impact Factor 0.821. 